\title{
Crystallization of mixed rare earth (didymium) molybdates in silica gel
}

\author{
SANJAY PANDITA, RAVENDER TICKOO, K K BAMZAI, P N KOTRU* and \\ NEERA SAHNI ${ }^{\dagger}$
}

Department of Physics, University of Jammu, Jammu 180 006, India

${ }^{\dagger}$ Department of Geology, Panjab University, Chandigarh 160 014, India

MS received 3 July 2000; revised 2 May 2001

\begin{abstract}
Experiments on the growth of mixed rare earth (didymium-a combination of La, Nd, Pr and Sm) molybdates in silica gel medium are reported. The optimum conditions conducive for the growth of these crystals are described and discussed. Concentration programming is reported to enhance the size of crystals by two-fold; the maximum size obtained being about $1 \mathrm{~mm}^{3}$. EDAX results suggest the crystals to be heptamolybdates of type $\mathrm{R}_{2} \mathrm{Mo}_{7} \mathrm{O}_{24}$, bearing composition $\mathrm{La}_{1.23} \mathrm{Nd}_{0.43} \mathrm{Pr}_{0.29} \mathrm{Sm}_{0.05} \mathrm{Mo}_{7} \mathrm{O}_{24}$. The didymium molybdate crystals assume morphologies corresponding to those of spherulites, platelets, cuboids and coalesced crystals. Twinned structure in didymium molybdate crystals are also reported. It is explained that spherulitic morphologies result from aggregates of crystals joining in a spherical envelope. It is suggested that the crystals of didymium molybdates grow by two-dimensional spreading and piling up of layers.
\end{abstract}

Keywords. Crystal growth; didymium molybdate; gel method; morphology; EDAX.

\section{Introduction}

Growth of mixed crystals is interesting because their characteristics change from those of the crystals of single components grown separately. For example, mixed crystals are found to be harder than pure ones (Dharma Prakash and Rao 1987; Kotru et al 1989a). Large variations in the hardness of pure and mixed $\mathrm{KBr}$ and $\mathrm{KI}$ system are reported (Subba Rao and Babu 1978, 1980). Gits et al (1985) reported doping effect on the quality of ADP when the same was doped with chromium. The effect of doping on gel grown crystals was investigated by Dishovsky and MLadenova (1981) and Dennis and Henisch (1967). Significant changes in physico-chemical characteristics of mixed crystals as brought about in comparison with those of the pure ones were reported in other cases as well (Belikova and Belyaev 1962; Klevtsov et al 1975; Kotru et al 1989b). Growth of mixed crystals by gel method was reported by Patel and Arora (1977a, b), Wiktorowska et al (1983), Kotru and Raina (1986) and Jain et al (1996). Growth of neodymiumlanthanum heptamolybdate crystals in silica gels was reported by Bhat et al (1995). Rare earth molybdates, in general, have the potential of wide application in science and technology on account of the fact that they exhibit interesting fluorescent, laser, piezoelectric, ferroelectric and ferroelastic properties. Rare earth molybdates are

\footnotetext{
*Author for correspondence
}

accepted as being ferroelectric (Borchardt and Bierstedt 1996). We have succeeded in growing single crystals of praseodymium molybdate in silica gels in our laboratory (Pandita et al 1998).

In this paper we report a method of growing mixed rare earth (didymium) molybdate crystals at room temperature by encapsulating the suitable reactants in a suitable network employing the gel method. The method involves the growth of didymium (a mixture of $\mathrm{La}, \mathrm{Nd}, \mathrm{Pr}$ and $\mathrm{Sm}$ ) molybdate crystals by controlled diffusion of chemical reagents through the medium of silica gel. The gel medium provides a three-dimensional structure in which the crystal nuclei are directly held in position for their formation and development, without generating convection currents and turbulence and by maintaining chemical inertness. A literature survey unfolds that no work has so far been reported on the growth of mixed crystals of La, $\mathrm{Nd}$, Pr and Sm (didymium) molybdates in sodium silicate gels at room temperature. The work was undertaken to obtain single crystals of mixed rare earth molybdates for scientific investigations leading to an understanding of their characteristic properties, including dielectric, mechanical, structural and thermal. In this paper the results of experiments on crystallization of didymium molybdate is presented.

\section{Experimental}

A single tube of dimensions $2.5 \mathrm{~cm}$ diameter and $20 \mathrm{~cm}$ length, with $\sim 75 \%$ of its volume filled by one type of 
reactant (called 'lower reactant' (LR)) encapsulated in the silica gel network and $\sim 25 \%$ filled up with the other reactant (didymium chloride) (known as the upper reactant (UR)), was used as a crystallizer as schematically shown in figure 1.

The process of preparation was as follows: $284.20 \mathrm{~g}$ of $\mathrm{Na}_{2} \mathrm{SiO}_{3} \cdot 9 \mathrm{H}_{2} \mathrm{O}$ was dissolved in 11 of distilled water in order to obtain a solution of concentration $1 \mathrm{M}$. The solution was left undisturbed for a few days and a clear solution was obtained on decantation. The second solution of lower reactant was prepared by adding ammonium molybdate and ammonium nitrate, each weighing $10 \mathrm{~g}$, to $65 \mathrm{cc}$ of distilled water. This solution was thoroughly mixed with a magnetic stirrer and then $30 \mathrm{cc}$ of conc. $\mathrm{HNO}_{3}$ was added to it drop by drop till a white precipitate was formed. At this stage $30 \mathrm{cc}$ of distilled water was added in order to obtain $125 \mathrm{cc}$ of lower reactant of $0.06 \mathrm{M}$. The first solution (sodium metasilicate solution) was then mixed with the second solution (lower reactanta source of molybdenum ions) in the crystallizer. The combined solution of the desired $\mathrm{pH}$ was allowed to set in the crystallizer and then aged for the desired time. In our experiments we used gels of ages $24,48,72 \mathrm{~h}$ or even more. After the gel was set and aged, the upper reactant of a desired molarity was poured along the sides of the tube, ensuring that this process does not break the gel. All experiments were conducted at room temperature of about $25^{\circ} \mathrm{C}$. Figure 2 represents the growth of didymium molybdate crystals in a crystallizer. The crystals were examined under optical microscopes, Epignost (for rapid visual examination) and metallurgical microscope (Neophot-2), of Carl Zeiss, Germany and scanning electron

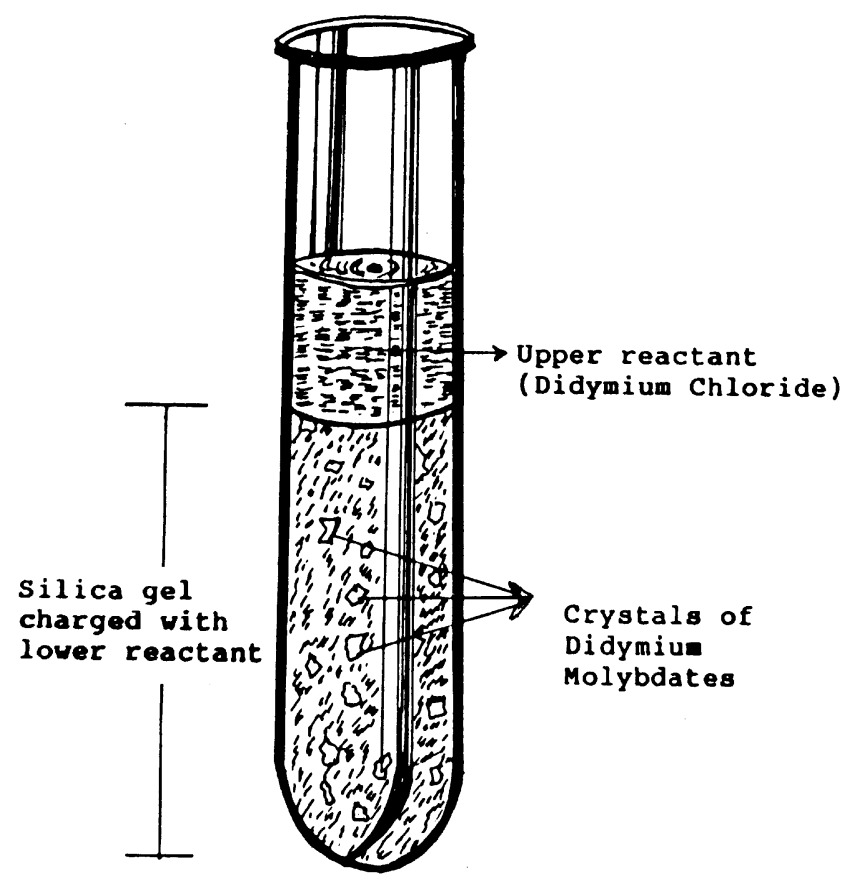

Figure 1. Schematic diagram of a crystallizer. microscope (model JEOL JSM-25 S) coupled with the EDAX system model KEVEX Delta Class. Before examination under the SEM, the crystals were coated with gold by ion-sputter coater model JFC-1100 (JEOL) in order to provide even granularity and efficient high reproducibility.

\section{Results and discussion}

In order to establish optimum conditions conducive for the growth of mixed rare earth didymium molybdates in the form of single crystals of size suitable for scientific studies, experiments were performed under different conditions of growth parameters including gel concentration, gel $\mathrm{pH}$, concentration of upper and lower reactants and gel ageing. Table 1 provides summary of various details of the experiments and the results obtained. The main conclusion of these experiments was that the optimum conditions for the growth of better size and quality of crystals are gel $\mathrm{pH} 4.5$; UR concentration, $0.25 \mathrm{M}$; LR concentration (preparation of ammonium paramolybdate, ammonium nitrate and nitric acid), 0.03 M; gel ageing, $72 \mathrm{~h}$ and gel concentration, $0.25 \mathrm{M}$. In an effort to grow crystals of size bigger than what was obtained from the above optimum conditions, experiments on concentration

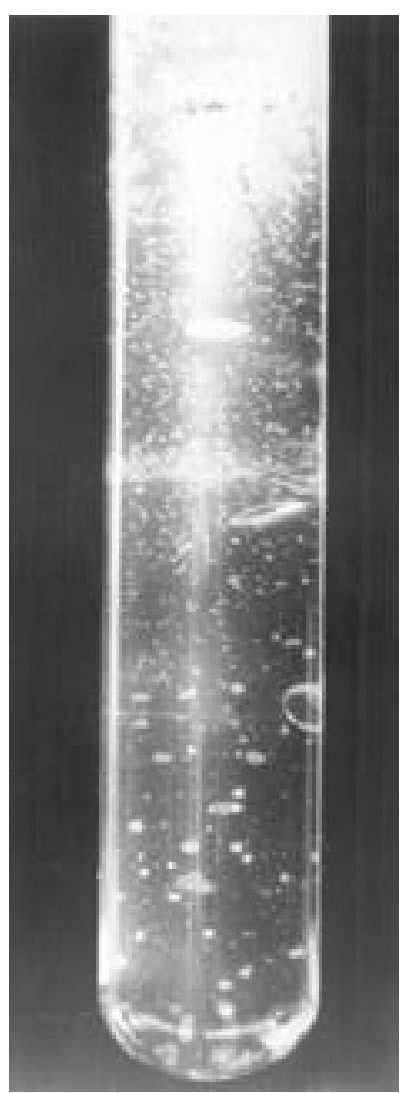

Figure 2. Growth of didymium molybdate in a crystallizer. 
Table 1. Details of experiments used for the growth of didymium molybdate crystals.

\begin{tabular}{|c|c|c|c|}
\hline Experiment & Constant parameters & Changing parameters & Results \\
\hline Variation of gel ageing & $\begin{array}{l}\text { UR conc. }(0 \cdot 25 \mathrm{M}) \\
\text { LR conc. }(0 \cdot 03 \mathrm{M}) \\
\text { Gel pH }(4 \cdot 5) \\
\text { Gel conc. }(0 \cdot 5 \mathrm{M})\end{array}$ & $\begin{array}{l}\text { Gel ageing } \\
(24,48,72,96,144 \mathrm{~h})\end{array}$ & 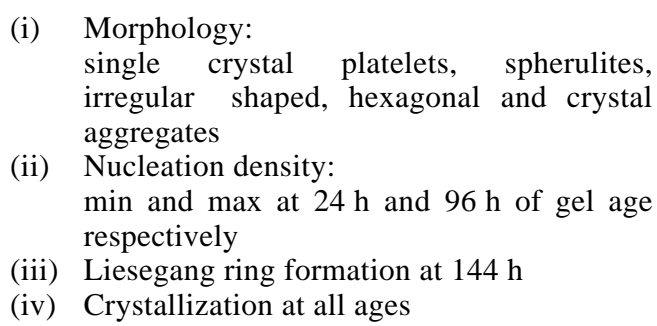 \\
\hline Variation of $\mathrm{pH}$ & $\begin{array}{l}\text { UR conc. }(0.25 \mathrm{M}) \\
\text { LR conc. }(0 \cdot 03 \mathrm{M}) \\
\text { Gel age } 72 \mathrm{~h} \\
\text { Gel conc. }(0.5 \mathrm{M})\end{array}$ & $\begin{array}{l}\text { Gel pH } \\
(4,4 \cdot 5,5,5 \cdot 5,6,6 \cdot 5)\end{array}$ & 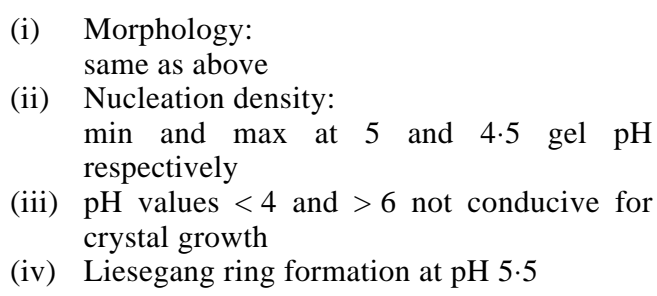 \\
\hline Variation of LR & $\begin{array}{l}\text { UR conc. }(0 \cdot 25 \mathrm{M}) \\
\text { Gel age } 72 \mathrm{~h} \\
\text { Gel pH }(4 \cdot 5) \\
\text { Gel conc. }(0 \cdot 5 \mathrm{M})\end{array}$ & $\begin{array}{l}\text { LR conc. } \\
(0.015,0.03,0.06 \mathrm{M})\end{array}$ & $\begin{array}{ll}\text { (i) } & \text { Morphology: } \\
& \text { same as above } \\
\text { (ii) Nucleation density: } & \\
& \text { min and max at } 0.015 \mathrm{M} \text { and } 0.06 \mathrm{M} \text { of } \\
& \text { LR respectively } \\
\text { (iii) } & \text { Crystals nucleate at all LR conc. }\end{array}$ \\
\hline Variation of UR & $\begin{array}{l}\text { LR conc. }(0 \cdot 06 \mathrm{M}) \\
\text { Gel age } 72 \mathrm{~h} \\
\text { Gel pH }(4 \cdot 5) \\
\text { Gel conc. }(0.5 \mathrm{M})\end{array}$ & $\begin{array}{l}\text { UR conc. } \\
(0 \cdot 25,0 \cdot 5,0 \cdot 75,1 \mathrm{M})\end{array}$ & $\begin{array}{ll}\text { (i) } & \text { Morphology: } \\
& \text { same as above } \\
\text { (ii) } & \text { Nucleation density: } \\
& \text { min and max at } 0.5 \mathrm{M} \text { and } 0 \cdot 25 \mathrm{M} \text { of UR } \\
& \text { respectively } \\
\text { (iii) } & \begin{array}{l}\text { Isolated nuclei formed at } 0.25 \mathrm{M} \text { of UR } \\
\text { conc. } \\
\text { (iv) }\end{array} \\
\text { Crystals nucleate at all UR conc. }\end{array}$ \\
\hline Variation of gel conc. & $\begin{array}{l}\text { UR conc. }(0 \cdot 5 \mathrm{M}) \\
\text { Gel age } 72 \mathrm{~h} \\
\text { Gel pH }(4 \cdot 5) \\
\text { LR conc. }(0 \cdot 03 \mathrm{M})\end{array}$ & $\begin{array}{l}\text { Gel conc. } \\
(0.125,0.25,0.5 \mathrm{M})\end{array}$ & $\begin{array}{l}\text { Morphology: } \\
\text { same as above } \\
\text { Nucleation density: } \\
\text { min and max at } 0.25 \mathrm{M} \text { and } 0.5 \mathrm{M} \text { of gel } \\
\text { conc. respectively } \\
\text { Bigger crystal size at } 0.025 \mathrm{M} \\
\text { Crystals nucleate at all gel conc. }\end{array}$ \\
\hline
\end{tabular}

Abbreviations used: Upper reactant (didymium chloride) (UR); lower reactant (gel charged with ammonium molybdate and ammonium nitrate) (LR); dimensions of crystallizer and gel column in all experiments are $2.5 \mathrm{~cm}$ dia and $20 \mathrm{~cm}$ ht and $13 \mathrm{~cm}$ respectively.

programming were performed. In such experiments, $0 \cdot 5 \mathrm{M}$ of upper reactant was poured initially over gel (gel concentration $0.5 \mathrm{M}$ and gel age $72 \mathrm{~h}$ ) charged with lower reactant (ammonium molybdate and ammonium nitrate) of conc. $0.6 \mathrm{M}$. After every $144 \mathrm{~h}$, the upper reactant was drained off by a dropper and replaced by upper reactant of higher concentration of molarities viz. $1 \mathrm{M}, 1.5 \mathrm{M}, 2 \mathrm{M}$, $2.5 \mathrm{M}, 3 \mathrm{M}, 3.5 \mathrm{M}$ and $4 \mathrm{M}$, each time keeping all other growth parameters constant. It was observed that concentration programming led to crystals of increased size, enhancing the ultimate size to $1 \mathrm{~mm}^{3}$ which is more than double the maximum size obtained by normal methods of growth. It was further observed that concentration pro- gramming only influenced the size but not the morphology of the crystals. It takes about one month for the crystals to grow to the maximum possible size, whether the experiments are performed under normal process of growth or concentration programming. In order to verify if the starting composition and the consequent reaction through diffusion, as controlled by the silica gel, yields crystals of the expected composition, both the starting composition of UR as well as the crystals were examined by EDAX. Figures 3 and 4 show the EDAX patterns obtained from the UR (didymium chloride) and the crystals respectively. The EDAX of figure 3 clearly shows that the upper reactant (didymium chloride (UR)) does 


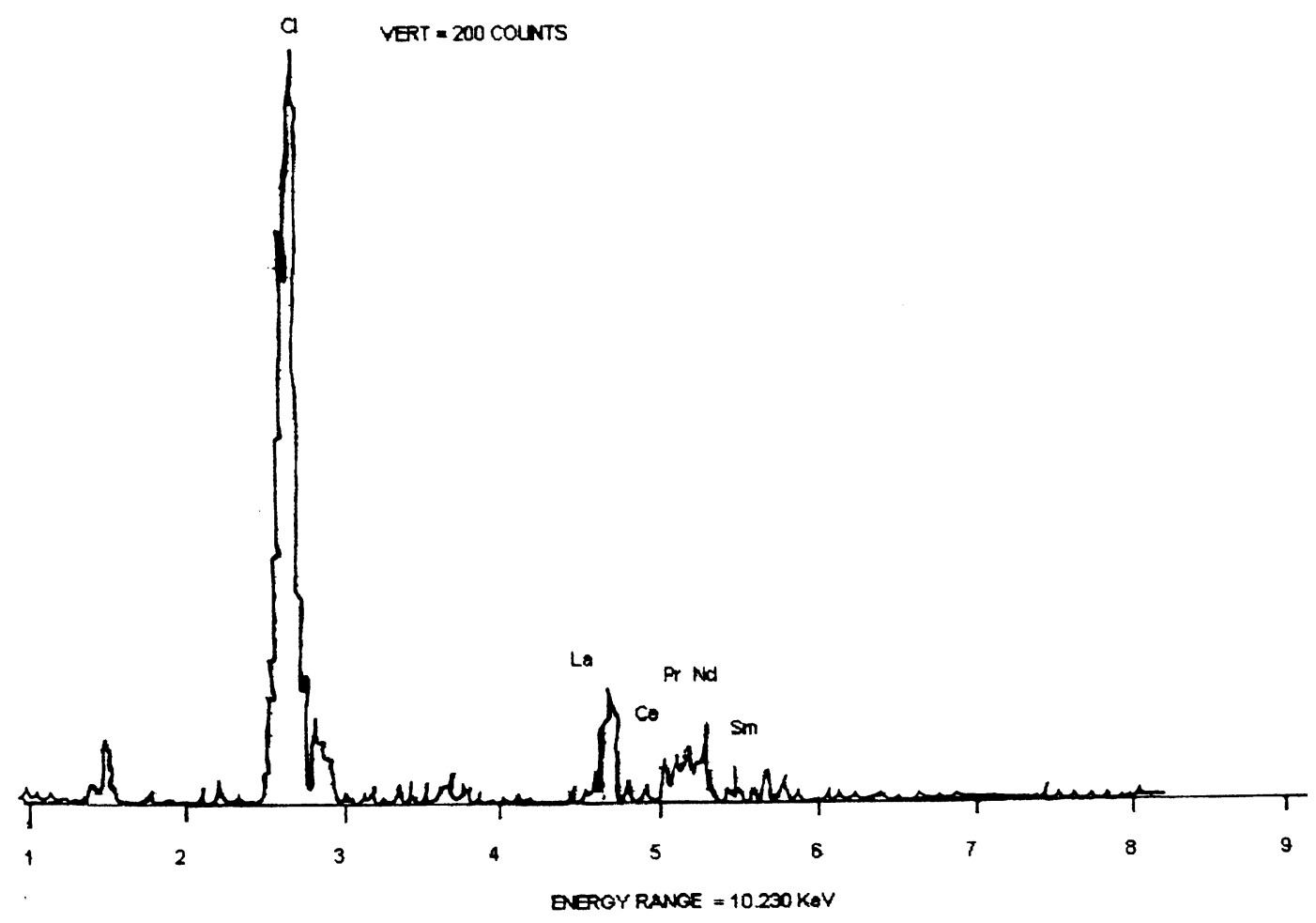

Figure 3. EDAX of starting composition (didymium chloride).

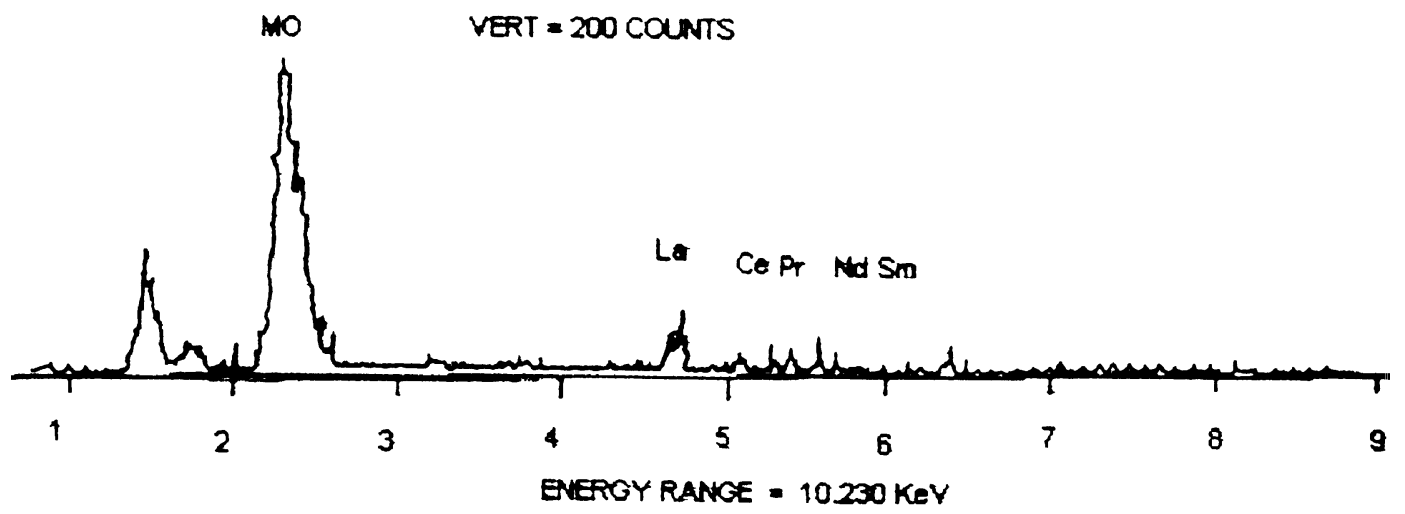

Figure 4. EDAX of didymium molybdate showing peaks corresponding to expected elements Mo, La, $\mathrm{Pr}, \mathrm{Nd}$ and $\mathrm{Sm}$.

Table 2. Data from EDAX analysis of starting material (didymium chloride).

\begin{tabular}{|c|c|c|c|c|c|c|c|c|c|c|c|c|}
\hline & \multicolumn{6}{|c|}{ Atomic percentage (at.\%) } & \multicolumn{6}{|c|}{ Weight percentage (wt $\%$ ) } \\
\hline & $\mathrm{La}$ & $\mathrm{Nd}$ & $\operatorname{Pr}$ & $\mathrm{Sm}$ & $\mathrm{Ce}$ & $\mathrm{Cl}$ & $\mathrm{La}$ & $\mathrm{Nd}$ & $\operatorname{Pr}$ & $\mathrm{Sm}$ & $\mathrm{Ce}$ & $\mathrm{Cl}$ \\
\hline Experimental & $8 \cdot 17$ & $5 \cdot 11$ & $4 \cdot 09$ & $1 \cdot 50$ & $0 \cdot 25$ & $80 \cdot 9$ & $20 \cdot 3$ & $13 \cdot 2$ & $10 \cdot 3$ & $4 \cdot 06$ & 0.64 & 51.4 \\
\hline Calculated & $8 \cdot 60$ & $5 \cdot 20$ & $4 \cdot 20$ & $1 \cdot 60$ & $0 \cdot 40$ & $80 \cdot 0$ & $21 \cdot 0$ & $13 \cdot 2$ & $10 \cdot 4$ & $4 \cdot 24$ & $0 \cdot 98$ & $50 \cdot 2$ \\
\hline
\end{tabular}

contain the ions of four rare earths including $\mathrm{La}, \mathrm{Nd}, \mathrm{Pr}$ and $\mathrm{Sm}$ with traces of Ce. Table 2 gives the atomic and weight per cent of the contents of the UR. The EDAX of figure 4 shows peaks corresponding to all the four major rare earths $\mathrm{La}, \mathrm{Nd}, \mathrm{Pr}$ and $\mathrm{Sm}$ in the grown crystals of didymium molybdate. Table 3 gives the experimentally and theoretically calculated atomic and weight per cent of the rare earths present in the crystal. The agreement between the experimental quantitative estimation and theoretically calculated values suggest the molecular 
Table 3. Data from EDAX analysis of $\mathrm{Di}_{2} \mathrm{Mo}_{7} \mathrm{O}_{24}$.

\begin{tabular}{|c|c|c|c|c|c|c|c|c|c|c|}
\hline & \multicolumn{5}{|c|}{ Atomic percentage (at.\%) } & \multicolumn{5}{|c|}{ Weight percentage (wt\%) } \\
\hline & $\mathrm{La}$ & $\mathrm{Nd}$ & $\operatorname{Pr}$ & $\mathrm{Sm}$ & Mo & $\mathrm{La}$ & $\mathrm{Nd}$ & $\operatorname{Pr}$ & $\mathrm{Sm}$ & Mo \\
\hline Experimental & $9 \cdot 71$ & $3 \cdot 40$ & $2 \cdot 29$ & $0 \cdot 39$ & $84 \cdot 2$ & $13 \cdot 1$ & $4 \cdot 76$ & $3 \cdot 13$ & 0.57 & $78 \cdot 4$ \\
\hline Calculated & $13 \cdot 6$ & $4 \cdot 78$ & $3 \cdot 22$ & $0 \cdot 55$ & $77 \cdot 8$ & $17 \cdot 9$ & $6 \cdot 51$ & $4 \cdot 29$ & 0.79 & $70 \cdot 5$ \\
\hline
\end{tabular}

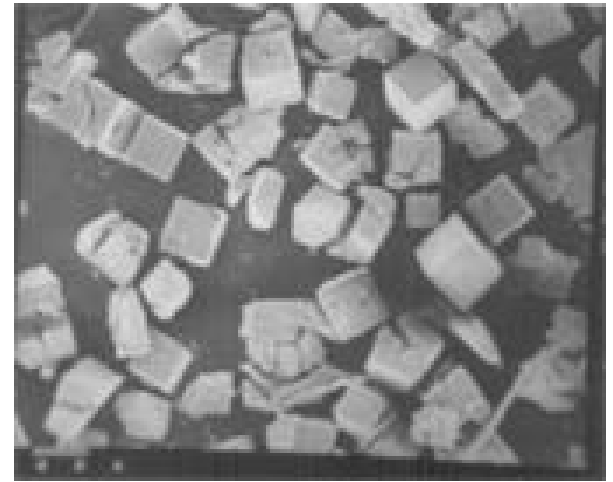

Figure 5. Different morphologies of didymium molybdates.

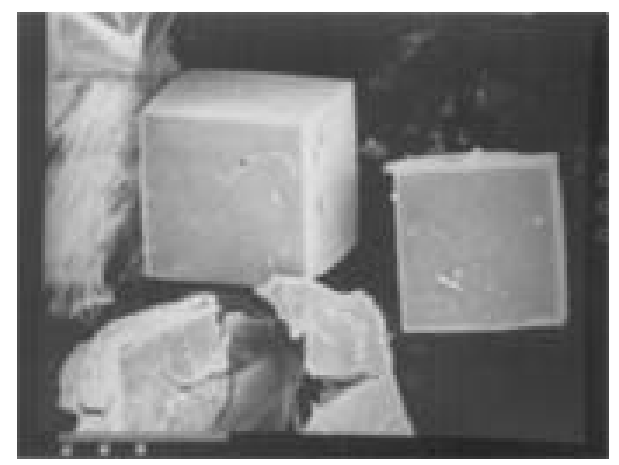

Figure 6. A regularly facetted crystal platelet with its plane faces.

formula of the grown crystal to be $\mathrm{La}_{1 \cdot 23} \mathrm{Nd}_{0.43} \mathrm{Pr}_{0.29}$ $\mathrm{Sm}_{0.05} \mathrm{Mo}_{7} \mathrm{O}_{24}$. The EDAX did show some traces of Ce but the quantity is of negligible amount which was ignored. The proposed molecular formula of the crystals grown suggests it to be didymium heptamolybdate of the type $\mathrm{R}_{2} \mathrm{Mo}_{7} \mathrm{O}_{24}$.

It is interesting to have a look at the morphology of mixed crystals obtained by the crystal growth method adopted here. The morphology of the crystals is described here.

Figure 5 shows different types of morphologies of didymium molybdates as viewed under scanning electron microscope. The various types of morphologies include spherulites, platelets, cuboids and coalesced crystals. It is interesting to see that most of the crystal surfaces are plane, devoid of any growth structures on them. Figure 6 shows regularly faceted crystal platelet with its plane

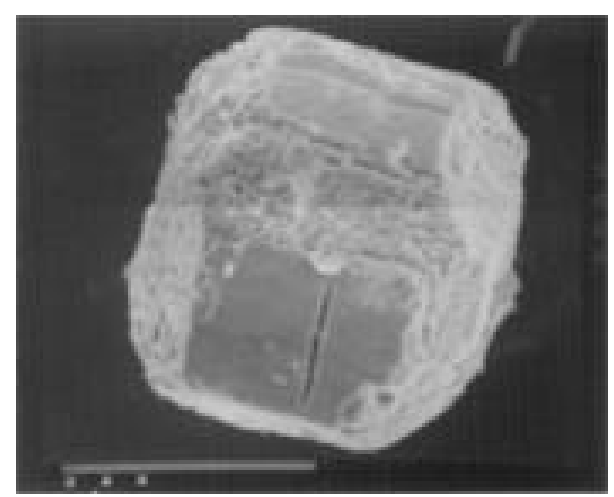

Figure 7. A crystal exhibiting layered growth.

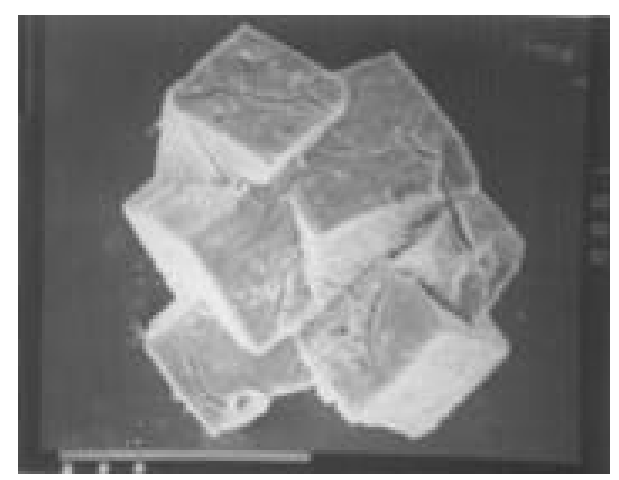

Figure 8. Twinned structure of didymium molybdates.

faces. A crystal exhibiting layered growth is shown in figure 7. One may notice that these crystals develop cracks produced due to vacuum coating or electron beam heating under the SEM which suggests the crystals are thermally unstable and may be carrying water of hydration. The details of their thermal behaviour as revealed by thermoanalytical studies conducted on them by TGA/ DTA/DSC techniques are being worked out and will be published elsewhere. Sometimes twinned crystals are also obtained. Figure 8 is an example of twinned structures in didymium molybdates.

\section{Conclusions}

From the above described experiments and results, the following conclusions are drawn: 
(I) Employing single gel single tube crystallizer, and using didymium chloride as the upper reactant and a solution prepared from $\left(\mathrm{NH}_{4}\right)_{6} \mathrm{Mo}_{7} \mathrm{O}_{24}-\mathrm{NH}_{4} \mathrm{NO}_{3}-\mathrm{HNO}_{3}$ as the lower reactant impregnated in sodium meta silicate gel, leads to crystallization of didymium (a mixture of four rare earths $\mathrm{La}, \mathrm{Nd}, \mathrm{Pr}$ and $\mathrm{Sm}$ ) heptamolybdate, bearing composition $\mathrm{La}_{1 \cdot 23} \mathrm{Nd}_{0.43} \mathrm{Pr}_{0 \cdot 29} \mathrm{Sm}_{0.05} \mathrm{Mo}_{7} \mathrm{O}_{24}$.

(II) The gel grown didymium heptamolybdate crystals assume varied morphologies including spherulites, cuboids, platelets, multifaceted and coalesced crystals. Twinned structures are also formed.

(III) The crystals grow mainly by two-dimensional spreading and piling up of layers, suggesting growth of crystals at high supersaturation.

\section{References}

Bhat S, Kotru P N and Kaul M L 1995 Mater. Sci. \& Engg. B34 138

Belikova G S and Belyaev I M 1962 Growth of crystals (eds) A V Shubnikov and N N Sheftal (New York: Consultants Bureau) 3 p. 228

Borchardt H J and Bierstedt P E 1966 Appl. Phys. Lett. 850

Dennis J and Henisch H K 1967 J. Electrochem. Soc. 114263
Dharma Prakash S M and Rao Mohan 1987 Cryst. Res. Technol. 221095

Dishovsky N and Boncheva Z-MLadenova 1981 J. Cryst. Growth 51147

Gits S, Robert M C and Lefaucheuse L 1985 J. Cryst. Growth $\mathbf{7 1} 203$

Jain A, Kotru P N and Kaul M L 1996 J. Mater. Sci. Technol. 1281

Klevtsov P V, Kozeeva C P, Klevtsova R F and Novgorodtseva N A 1975 Growth of crystals (eds) N N Sheftal and E I Givargizov (New York: Consultants Bureau) 9107

Kotru P N and Raina K K 1986 J. Mater. Sci. Lett. 5760

Kotru P N, Razdan A K and Wanklyn B M 1989a J. Mater. Sci. 242401

Kotru P N, Razdan A K and Wanklyn B M 1989b J. Mater. Sci. 24793

Pandita S, Hangloo V, Bamzai K K, Kotru P N and Sahni Neera 1998 Semiconductor materials: Characterization and device applications (ed.) R K Bedi (Amritsar: GND Univ.)

Patel A R and Arora S K 1977a J. Cryst. Growth 37343

Patel A R and Arora S K 1977b Krist. Tech. 13343

Subba Rao U V and Babu Hari 1978 Pramana-J. Phys. 11 147

Subba Rao U V and Babu Hari 1980 Indian J. Phys. A54 147

Wiktorowska B, Borecka B and Karniewicz J 1983 J. Mater. Sci. 18416 